\title{
Eficiência de duas técnicas de recuperação de espermatozoides epididimários de cães e avaliação seminal pós-criopreservação
}

\author{
[Efficiency of two techniques for recovering epididymal spermatozoids from dogs \\ and seminal evaluation post cryopreservation] \\ S.T.P. Lopes ${ }^{1}$, M.A.C. Sousa Filho ${ }^{1}$, J.H.L. Silva ${ }^{1}$, F.N. Barros ${ }^{1}$, M.A. Castelo Branco ${ }^{2}$, \\ L.S. Melo Evangelista ${ }^{3}$, J.A.T. Souza ${ }^{4}$ \\ ${ }^{1}$ Aluno de pós-graduação - Centro de Ciências Agrárias - Universidade Federal do Piauí - Teresina, PI \\ ${ }^{2}$ Centro Universitário Maurício de Nassau - Teresina, PI \\ ${ }^{3}$ Centro de Ciências da Saúde - Universidade Federal do Piauí - Teresina, PI \\ ${ }^{4}$ Centro de Ciências Agrárias - Universidade Federal do Piauí - Teresina, PI
}

\begin{abstract}
RESUMO
O objetivo deste trabalho foi avaliar a recuperação de espermatozoides epididimários de cães castrados, utilizando as técnicas de fluxo retrógrado (FR) e flutuação (FL) em diluidor Tris-gema, antes e após a criopreservação. Foram coletados 30 complexos testículo-epididímos (CTE), sendo 15 para FR e 15 para FL, e, logo após a recuperação dos espermatozoides, foram analisadas as alterações morfológicas nessas células espermáticas. Após a adição do diluidor, foram avaliados os parâmetros de motilidade total (MOT) e vigor (V) espermáticos. O sêmen pós-criopreservado foi submetido ao teste de termorresistência nos tempos T0, T30, T60 e T90 minutos, além da avaliação das membranas plasmática e acrossomal por sondas fluorescentes. Não houve diferença estatística entre as técnicas quanto à MOT e ao vigor no sêmen diluído (FR-MOT: 82,3\% e V: 3,4; FL-MOT: 79,6\% e V: 3,2) e pós-criopreservado (FR-MOT: 34\% e V: 2,8; FLMOT: $30 \%$ e V: 2,7). A partir do T30, houve diferença significativa quanto à MOT e ao vigor nas técnicas utilizadas, e o tempo também prejudicou o acrossoma espermático a partir do T30. Conclui-se que as técnicas de recuperação de espermatozoides epididimários de cães castrados, testadas neste trabalho, podem ser utilizadas para refrigeração e criopreservação de sêmen.
\end{abstract}

Palavras-chave: canino, epidídimo, flutuação, fluxo retrógrado, sêmen

\begin{abstract}
The objective of this work was to evaluate the recovery of epididymal spermatozoa from castrated dogs using retrograde flow (FL) and flotation (FL) techniques in Tris-egg yolk diluent, before and after cryopreservation. Thirty testicle-epididymal complexes (CTE) were collected, 15 for FR and 15 for FL and soon after spermatozoid recovery, morphological changes in these spermatic cells were analyzed. After addition of the diluent, the parameters of total motility (MOT) and vigor $(V)$ were evaluated. The postcryopreserved semen was submitted to thermoresistance (TTR) test at T0, T30, T60 and T90 minutes, as well as the plasma and acrosomal membrane evaluation by fluorescent probes. There was no statistically significant difference between techniques tested for MOT and vigor in the diluted semen (FR-MOT: 82.3\% and V: 3.4, FL-MOT: $79.6 \%$ and $V: 3.2$ ) and post-cryopreserved (FR-MOT: $34 \%$ and V: 2.8, FL-MOT: $30 \%$ and V: 2.7). From the T30 there was a significant difference regarding MOT and vigor in the used techniques, and the time also damaged the spermatic acrosome from the T30. It is concluded that the epididymal spermatozoa recovering techniques from castrated dogs, tested in this study, can be used for semen refrigeration and cryopreservation.
\end{abstract}

Keywords: canine, epididymis, flotation, retrograde flow, semen

Recebido em 10 de setembro de 2018

Aceito em 11 de fevereiro de 2020

E-mail: sabrina.pereiralopes@hotmail.com 


\section{INTRODUÇÃO}

A coleta de espermatozoides epididimários é utilizada, experimentalmente, em diversas espécies de animais domésticos (Granemann, 2006; Mota Filho et al., 2013; Lima et al., 2016; Benítez et al., 2018) e silvestres (Martinez-Pastor et al., 2006), sendo um recurso importante em casos de criação de bancos de germoplasma de espécies de animais em perigo de extinção e de conservação de animais domésticos de alto valor zootécnico (Benítez et al., 2018) ou de grande estima, que precisam ser esterilizados ou que vêm a óbito.

As técnicas utilizadas possibilitam, dentro de um determinado período de tempo, a recuperação dos espermatozoides da cauda do epidídimo do animal, podendo assegurar a preservação do material genético (Mota Filho e Silva, 2012), já que esses gametas, assim que recolhidos, são capazes de resistir à refrigeração, até serem, posteriormente, criopreservados e comercializados (Martins, 2007).

A obtenção de espermatozoides epididimários em humanos e animais pode ser facilitada por diversas técnicas, sendo preferencialmente realizada por duas: a técnica de flutuação e a de fluxo retrógrado. A primeira consiste em fatiar a cauda do epidídimo e deixá-lo em um meio diluidor para que os espermatozoides migrem para o meio (Yu e Leibo, 2002; Ponglowhapan et al., 2006; Bergo, 2018), e a outra promove um fluxo retrógrado na cauda do epidídimo aplicando-se pressão aos vasos deferentes até que o conteúdo seminal da cauda saia através de um corte feito na junção com o corpo do epidídimo (Martinez-Pastor et al., 2006; Batista et al., 2016; Bustamante, 2019).

Na técnica de flutuação, o complexo testículoepidídimo é lavado e dissecado até o isolamento completo da cauda do epidídimo e do ducto deferente; estes são posicionados sobre uma placa de Petri com um diluidor aquecido a $37^{\circ} \mathrm{C}$. Em seguida, o fatiamento do epidídimo é realizado e a solução obtida é recuperada e avaliada (Yu e Leibo, 2002). Apesar de ser a técnica mais disseminada entre os pesquisadores, as amostras seminais tendem a se contaminar com os detritos celulares (Hori et al., 2015). Já a técnica de fluxo retrógrado tem uma melhor indicação, pois as amostras obtidas apresentam um menor nível de contaminação e são de melhor qualidade e rendimento em relação aos outros métodos (Martinez-Pastor et al., 2006; Bergo, 2018).

Nos diluidores seminais aplicados à reprodução canina, a gema de ovo é comumente utilizada por apresentar funções de proteção às células espermáticas durante as etapas de congelação e descongelação, com ação principal sobre a membrana plasmática dos espermatozoides (Hewitt et al., 2001). Além disso, sua utilização, juntamente com crioprotetores associados, constitui a melhor alternativa para a criopreservação do sêmen canino (Rizzoto et al., 2014).

É sabido que a criopreservação de espermatozoides recuperados da cauda do epidídimo de animais pode provocar mais danos às células espermáticas do que na congelação de espermatozoides colhidos por meio das técnicas convencionais, principalmente em se tratando de resistência ao choque térmico (Álvarez et al., 2012). Com isso, os métodos utilizados para a avaliação seminal pós-criopreservação devem ser precisos e eficazes.

Nesse contexto, este trabalho objetivou avaliar a taxa de recuperação de espermatozoides da cauda do epidídimo de cães, após a orquiectomia, utilizando as técnicas de fluxo retrógrado e flutuação, bem como avaliar a viabilidade desses espermatozoides em meio diluidor Tris-gema após a criopreservação, podendo, assim, oferecer mais uma ferramenta de biotecnologia aplicada à espécie canina.

\section{MATERIAL E MÉTODOS}

Este trabalho foi apresentado e aprovado pelo Comitê de Ética no Uso de Animais da Universidade Federal do Piauí (CEUA/UFPI), sob o número de protocolo 304/17. O experimento foi realizado de julho a dezembro de 2017, no município de Teresina, Piauí. Os critérios de inclusão para avaliação da sanidade dos animais utilizados neste experimento foram estabelecidos por meio de exames andrológicos preconizados pelo CBRA (Manual... CBRA, 2013), além da realização de hemograma, bioquímica sérica e sorologia para leishmaniose visceral (LV) e brucelose. 
Neste experimento foram utilizados 30 complexos testículo-epididímos (CTE) de cães machos aparentemente saudáveis, sexualmente maduros, com idade entre dois e oito anos de idade, pesando entre cinco e 15 quilogramas $(\mathrm{kg})$, sem raça definida (SRD), sendo $15 \mathrm{CTE}$ desses animais direcionados para cada técnica de recuperação de espermatozoides epididimários (flutuação e fluxo retrógrado). As amostras sanguíneas dos 30 cães utilizados neste trabalho foram colhidas em tubos a vácuo com anticoagulante para realização de hemograma e sem anticoagulante para as análises de bioquímica sérica (ureia, creatinina, proteínas totais, albumina e globulina) e sorologia para LV e brucelose.

Os exames hematológicos e bioquímicos foram realizados no Laboratório de Patologia Clínica do Hospital Veterinário da Universidade Federal do Piauí (HVU/UFPI) e a sorologia para LV canina foi realizada no Laboratório de Parasitologia do Departamento de Parasitologia e Microbiologia da UFPI, utilizando-se o teste sorológico TRDPP $^{\circledR}$, kit comercial da Bio-Manguinhos, conforme as recomendações do fabricante.

Na sorologia para brucelose, foi utilizada a técnica de imunodifusão em gel de ágar (IDGA), realizada no Laboratório de Fisiopatologia da Reprodução da Fêmea, UFPI. Essa técnica consistiu no emprego de antígeno contendo proteínas e lipopolissacarídeos solúveis, extraídos da bactéria Brucella ovis, amostra Reo 198, produzido pelo Instituto de Tecnologia do Paraná $\left(\right.$ Tecpar $\left.^{\circledR}\right)$, e as provas foram realizadas de acordo com as recomendações do fabricante.

A coleta dos 30 CTE foi realizada após a orquiectomia de cães provenientes de cirurgias eletivas realizadas no HVU/UFPI e em quatro clínicas veterinárias particulares do município de Teresina, Piauí. O material coletado foi acondicionado em sacos plásticos estéreis, previamente identificados, contendo solução salina $(\mathrm{NaCl} 0,9 \%)$, e levado para o Laboratório de Biotecnologia da Reprodução Animal (LBRA/UFPI), até a realização do procedimento das técnicas, conforme metodologia proposta por Yo e Leibo (2002) e Mota Filho et al. (2014).

Na técnica de flutuação (FL), os epidídimos e os ductos deferentes foram dissecados até o isolamento completo da cauda do epidídimo e do ducto; estes foram posicionados sobre uma placa de Petri de 90x15mm de diâmetro e fatiados em cortes seriados, adicionados de $3 \mathrm{~mL}$ do diluidor Tris-gema aquecido a $37^{\circ} \mathrm{C}$, por 10 minutos. $\mathrm{Na}$ técnica de fluxo retrógrado (FR), após a dissecação do epidídimo, foi aplicada uma pressão nos vasos deferentes, por meio de uma lâmina de vidro, até que o conteúdo seminal da cauda do epidídimo saísse através de um corte realizado na junção com o corpo do epidídimo, sendo também colocados em placa de Petri, adicionados com a mesma quantidade e o mesmo diluidor supracitados.

O volume do sêmen recuperado por cada técnica e, posteriormente, diluído, foi mensurado em tubo de coleta do tipo Falcon de $15 \mathrm{~mL}$. Em seguida, foram avaliados os parâmetros de motilidade total (0-100\%), vigor espermático (1-5), concentração (espermatozoides/mL) e morfologia espermática (\%). A concentração espermática foi determinada pela técnica da Câmara de Neubauer; a morfologia espermática foi avaliada por meio da preparação úmida; e a leitura foi realizada sob microscopia de contraste de fases em aumento de 2000x. Nesta última, foram avaliados 200 espermatozoides por lâmina e classificados em defeitos maiores e defeitos menores (Blom, 1973; Manual... CBRA, 2013).

Logo após essa avaliação, os tubos de coleta com o sêmen diluído foram submetidos ao processo de refrigeração em geladeira, até se atingir a temperatura de $5^{\circ} \mathrm{C}$, sendo estabilizados por uma hora nessa temperatura. Em seguida, as amostras seminais foram envasadas em palhetas de $0,5 \mathrm{~mL}$, previamente identificadas, e colocadas a $5 \mathrm{~cm}$ da superfície de um isopor contendo nitrogênio líquido, durante cinco minutos, e posteriormente foram colocadas em botijão para criopreservação.

Após duas semanas desse procedimento, foi realizado o teste de termorresistência (TTR). Para tanto, as amostras de sêmen foram colocadas em microtubos de $1,5 \mathrm{~mL}$, incubadas a $37^{\circ} \mathrm{C}$, em banho-maria, e avaliadas quanto à motilidade total e ao vigor espermático, nos tempos T0, T30, T60 e T90 minutos, sob microscopia de luz em aumento de 2000x.

Foi avaliada a integridade da membrana plasmática espermática com o uso de sondas fluorescentes, conforme descrito por Harrison e Vickers (1990), em que alíquotas de $50 \mu \mathrm{L}$ de sêmen foram diluídas em $150 \mu \mathrm{L}$ de Tris contendo 
$5 \mu \mathrm{L}$ de diacetado de carboxifluoresceína) $(0,46 \mathrm{mg} / \mathrm{mL}$ em DMSO) e $20 \mu \mathrm{L}$ de iodeto de propídio $(0,5 \mathrm{mg} / \mathrm{mL}$ em PBS $)$, incubadas por 10 minutos a $37^{\circ} \mathrm{C}$ e fixadas com PBS contendo $0,5 \%$ de glutaraldeído. A análise ocorreu pela contagem de 200 espermatozoides em microscópio de epifluorescência, em aumento de 4000x. As células que apresentaram fluorescência verde foram consideradas com membrana plasmática íntegra, enquanto aquelas que apresentaram fluorescência vermelha foram consideradas danificadas.

Para análise do acrossoma, foi utilizado o isocianato de fluoresceína conjugado (FITCPNA). Uma alíquota de $100 \mu \mathrm{L}$ da solução de FITC-PNA $(1 \mathrm{mg} / \mathrm{mL})$ foi descongelada e adicionada a $900 \mu \mathrm{L}$ de PBS para se obter a concentração final de $100 \mu \mathrm{g} / \mathrm{mL}$. Alíquotas de $20 \mu \mathrm{L}$ dessa solução foram inseridas sobre lâminas previamente preparadas com $10 \mu \mathrm{L}$ de sêmen descongelado diluído em $990 \mu \mathrm{L}$ de $\mathrm{PBS}$, e incubadas por 15 minutos em câmara úmida a $4^{\circ} \mathrm{C}$, na ausência de luz. Imediatamente antes da avaliação, $5 \mu \mathrm{L}$ de meio de montagem $(4,5 \mathrm{~mL}$ de glicerol, 0,5mL de PBS, 5mg de fenilenodiamina, $5 \mathrm{mg}$ de azida sódica) foram colocados sobre uma lâmina e cobertos com lamínula. Foram avaliados 200 espermatozoides, em aumento de 10000x, usando microscopia de epifluorescência. As células espermáticas foram classificadas como portadoras de acrossomas intactos (AI), quando a região acrossomal apresentou fluorescência verde brilhante, e acrossoma reagido (AR), quando apresentou apenas na região equatorial da cabeça espermática a coloração verde ou fluorescência verde.

Para a análise estatística dos dados, foram obtidas as médias e o desvio-padrão das taxas de recuperação de espermatozoides epididimários, utilizando-se a análise de variância, Programa Statistical Analysis System for Windows (SAS), e empregando-se o teste de Tukey no caso de diferenças significativas entre as técnicas testadas. Os resultados foram considerados significativos quando $\mathrm{P}<0,05$.

\section{RESULTADOS E DISCUSSÃO}

Os animais deste trabalho encontravam-se dentro dos critérios de inclusão de sanidade preconizados para este estudo, com os perfis hematológicos e bioquímicos dentro da normalidade para a espécie canina e negativos para os testes sorológicos de LV e brucelose. De acordo com os resultados encontrados, foi possível confirmar que as técnicas de FL e FR são ferramentas de biotecnologia importantes que podem ser utilizadas para a recuperação de espermatozoides da cauda do epidídimo de cães após a orquiectomia, sendo relevante a avaliação das características espermáticas pré e pós-congelação.

Quanto à morfologia espermática, as amostras apresentaram índice elevado de anormalidades espermáticas, algumas chegando a $74,0 \%$ de defeitos maiores e 78,6\% de defeitos totais. Vale ressaltar que o epidídimo é o local em que os espermatozoides amadurecem, desenvolvem-se e ficam armazenados até a ejaculação, portanto a passagem das células espermáticas pelas diferentes partes desse órgão pode levar a mudanças e alterações importantes (Plante e Zeleznik, 2014), lembrando, ainda, que essas alterações morfológicas podem variar de acordo com a técnica de recuperação espermática utilizada.

Em alguns resultados, espermatozoides colhidos da cauda do epidídimo de cães, por meio da técnica de flutuação, apresentaram uma quantidade de defeitos totais em porcentagem maior que a aceitável para a espécie canina, variando de 47 a 52\% (Hishinuma e Sekine, 2003; Varesi et al., 2014). Alguns autores observaram melhores resultados de morfologia dos espermatozoides quando recuperados pela técnica de fluxo retrógrado e com o uso de meio diluidor comercial à base de Tris, Caniplus ${ }^{\circledR}$ (Paula et al., 2018) e Nutricell ${ }^{\circledR}$ (Bustamante, 2019). Neste trabalho, FR também foi a técnica em que se observou menor frequência de alterações morfológicas nas células espermáticas avaliadas, quando comparada com a técnica de FL. Outros autores, para diminuir a quantidade de defeitos totais, fizeram uso de fluido prostático canino nas amostras espermáticas epididimárias (Hori et al., 2015), porém essa diminuição não foi observada nos resultados citados por Bustamante (2019).

A alteração morfológica frequentemente observada foi a gota citoplasmática distal (72\%). Esse defeito normalmente é o mais encontrado em espermatozoides provenientes da cauda do epidídimo, mesmo que em menores porcentagens (Hori et al., 2015; Pennacchisavi et al., 2015), o que provavelmente está relacionado com o 
processo de maturação espermática, já que durante sua passagem pelo epidídimo é que o espermatozoide perde esse resquício citoplasmático (Axnèr et al., 1998).

A literatura relata que a coleta de espermatozoides da cauda do epidídimo por meio da técnica de fluxo retrógrado é a mais indicada (MartinezPastor et al., 2006), porém, neste estudo, não se obteve diferença significativa entre as técnicas avaliadas quanto aos parâmetros de motilidade e vigor, tanto no sêmen fresco diluído como no póscriopreservado (Tab. 1).

Tabela 1. Motilidade total e vigor de espermatozoides epididimários de cães orquiectomizados, obtidos por técnicas de recuperação espermática, avaliados no sêmen fresco diluído e criopreservado $(\chi \pm$ EMP)

\begin{tabular}{llccc}
\hline & \multicolumn{2}{c}{ Parâmetros } \\
\hline Técnicas & Sêmen fresco diluído & \multicolumn{2}{c}{ Sêmen criopreservado } \\
Flutuação & Motilidade & Vigor & Motilidade & Vigor \\
Fluxo retrógrado & $79,6 \pm 8,95 \mathrm{a}$ & $3,2 \pm 0,31 \mathrm{a}$ & $30 \pm 10,69 \mathrm{a}$ & $2,7 \pm 0,41 \mathrm{a}$ \\
\hline
\end{tabular}

Letras iguais na mesma coluna não indicam diferença estatística $(\mathrm{P}<0,05)$.

Em um estudo realizado com o objetivo de avaliar a qualidade seminal de cães, observou-se que a motilidade de espermatozoides recuperados pela técnica de FL foi de $65 \%$ (Yu e Leibo, 2002) e pela técnica de FR, em outro estudo, foi de $77 \%$ (Ponglowhapan et al., 2006), diferindo dos resultados encontrados neste trabalho, em que se observaram valores superiores de MOT no sêmen fresco diluído nas duas técnicas empregadas. Nota-se que, por meio da técnica de FR, outros trabalhos também apresentaram MOT superior a $80 \%$ em meio seminal diluído (Pennacchisavi et al., 2015; Bustamante, 2019). Esse parâmetro reflete a porcentagem de células espermáticas móveis e a qualidade do movimento dessas células, o que indica a viabilidade dos espermatozoides recuperados de epidídimos dos cães deste trabalho, diluídos em Tris-gema.

Em pesquisa realizada com amostras seminais recuperadas de epidídimos de cães, foi possível observar que, mesmo elas sendo mantidas sob refrigeração a $4^{\circ} \mathrm{C}$, por alguns dias, a motilidade espermática foi satisfatória, além da manutenção da integridade da membrana plasmática e da capacidade de se ligarem a zonas pelúcidas de oócitos (Yu e Leibo, 2002). Em outro estudo, a MOT de espermatozoides recuperados da cauda do epidídimo apresentou excelente qualidade por até $12 \mathrm{~h}$ sob refrigeração (Mota Filho et al., 2013). Entretanto, a viabilidade espermática pode diminuir conforme aumenta o tempo da retirada dos epidídimos e a recuperação dos espermatozoides da cauda desse órgão, além da temperatura em que os epidídimos devem ser mantidos da coleta até a refrigeração e criopreservação seminal (Bergstein-Galan et al., 2017).

O tempo em que os espermatozoides epididimários podem ficar expostos à temperatura ambiente e continuarem viáveis varia conforme a espécie animal. Em cães, os epidídimos podem ser conservados e os espermatozoides recuperados se colocados com maior brevidade possível sob temperatura de refrigeração; já quando mantidos em temperatura ambiente, não há muitos estudos sobre esse tempo de conservação.

Alguns trabalhos mostraram que, nos espermatozoides armazenados por até seis horas em temperatura de $20^{\circ} \mathrm{C}$, a MOT ainda é considerada favorável (Hori et al., 2009). Vale ressaltar que o tempo máximo da coleta dos CTE dos animais deste trabalho até o início dos procedimentos das técnicas de recuperação espermática girava em torno de duas horas, tempo considerado como aceitável para a conservação das características espermáticas para uso na congelação e após a descongelação (Hewitt et al., 2001).

Com relação à longevidade espermática após a criopreservação, no TTR, a partir do T30, houve influência negativa nos parâmetros de MOT e vigor, em ambas as técnicas, com redução considerável desses valores decorridos 30 minutos pós-criopreservação, não havendo diferença significativa entre as técnicas na análise desse parâmetro (Tab. 2 e 3). 
Eficiência de duas técnicas...

Tabela 2. Motilidade total de espermatozoides epididimários de cães orquiectomizados, obtidos por técnicas de recuperação espermática, avaliados por TTR, tempos T0 a T90 minutos pós-criopreservação $(\chi \pm$ EMP $)$

\begin{tabular}{lcccc}
\hline \multicolumn{4}{c}{ TTR (Motilidade) } \\
\hline Técnicas/Tempo & T0 & T30 & T60 & T90 \\
FL & $30 \pm 10,6 \mathrm{aA}$ & $20,1 \pm 11,1 \mathrm{bA}$ & $16,6 \pm 10,2 \mathrm{bA}$ & $8,3 \pm 10,6 \mathrm{cA}$ \\
FR & $34 \pm 12,9 \mathrm{aA}$ & $24,3 \pm 12,3 \mathrm{bA}$ & $16,3 \pm 10,2 \mathrm{bA}$ & $9,3 \pm 10,9 \mathrm{cA}$ \\
\hline
\end{tabular}

Letras minúsculas diferentes na mesma linha indicam diferença estatística dentro de cada técnica, de acordo com o tempo $(\mathrm{P}<0,05)$. Letras maiúsculas diferentes na mesma coluna indicam diferença estatística entre as técnicas, de acordo com o tempo $(\mathrm{P}<0,05)$.

Tabela 3. Vigor de espermatozoides epididimários de cães orquiectomizados, obtidos por técnicas de recuperação espermática, avaliados por TTR, tempos T0 a T90 minutos pós-criopreservação $(\chi \pm \mathrm{EMP})$

\begin{tabular}{lcccc}
\hline \multicolumn{5}{c}{ TTR (Vigor) } \\
\hline Técnicas/Tempo & T0 & T30 & T60 & T90 \\
FL & $2,73 \pm 0,40 \mathrm{aA}$ & $2,26 \pm 0,77 \mathrm{bA}$ & $2,03 \pm 0,87 \mathrm{bA}$ & $1,03 \pm 1,10 \mathrm{cA}$ \\
FR & $2,86 \pm 0,50 \mathrm{aA}$ & $2,43 \pm 0,79 \mathrm{bA}$ & $2,06 \pm 0,92 \mathrm{bA}$ & $1,01 \pm 1,10 \mathrm{cA}$ \\
\hline
\end{tabular}

Letras minúsculas diferentes na mesma linha indicam diferença estatística dentro de cada técnica, de acordo com o tempo $(\mathrm{P}<0,05)$. Letras maiúsculas diferentes na mesma coluna indicam diferença estatística entre as técnicas, de acordo com o tempo $(\mathrm{P}<0,05)$.

A ação do tempo sobre os parâmetros citados também foi observada em outro estudo, em que foram analisadas a MOT e o vigor espermático de amostras seminais pós-criopreservação de cães positivos e negativos para LV canina, nos tempos T0 a T60 minutos pós-criopreservação, observando-se também uma diminuição progressiva desses parâmetros após 30 minutos (Melo Evangelista et al., 2016).

A sobrevivência dos espermatozoides após os processos de congelação e descongelação aparentemente depende das características principais da membrana plasmática, como composição bioquímica, resistência física, comportamento térmico e osmótico (De Leeuw et al., 1991). Porém, acredita-se que a termorresistência pós-descongelação pode ser mais baixa para o espermatozoide canino do que para o de outras espécies, e esses resultados também podem ser atribuídos à variação de congelabilidade seminal individual de cada animal (Ström et al., 1997).

Durante a avaliação do acrossoma, o tempo prejudicou a integridade dessas células espermáticas também a partir do T30, diminuindo a quantidade de espermatozoides com acrossoma íntegro ao longo do tempo. Já na porcentagem de espermatozoides com membrana plasmática íntegra, o tempo não interferiu nessas células, mesmo decorridos 90 minutos póscriopreservação (Tab. 4 e 5). Entre as técnicas, não houve diferença significativa na avaliação dessas células.

Tabela 4. Porcentagem de membrana acrossomal íntegra dos espermatozoides epididimários de cães orquiectomizados, avaliados por sondas fluorescentes, tempos T0 a T90 minutos pós-criopreservação $(\chi$ \pm EMP)

\begin{tabular}{lcccc}
\hline & \multicolumn{4}{c}{ Integridade da membrana acrossomal (\%) } \\
\hline Técnicas/Tempo & T0 & T30 & T60 & T90 \\
FL & $26,8 \pm 18,6 \mathrm{aA}$ & $18,7 \pm 14,5 \mathrm{bA}$ & $13,6 \pm 13,4 \mathrm{bcA}$ & $9,2 \pm 8,5 \mathrm{cA}$ \\
FR & $20,4 \pm 15,5 \mathrm{aA}$ & $14,6 \pm 13,1 \mathrm{bA}$ & $9,8 \pm 9,2 \mathrm{bcA}$ & $7,7 \pm 7,8 \mathrm{cA}$ \\
\hline
\end{tabular}

Letras minúsculas diferentes na mesma linha indicam diferença estatística dentro de cada técnica, de acordo com o tempo $(\mathrm{P}<0,05)$. Letras maiúsculas diferentes na mesma coluna indicam diferença estatística entre as técnicas, de acordo com o tempo $(\mathrm{P}<0,05)$. 
Tabela 5. Porcentagem de membrana plasmática íntegra dos espermatozoides epididimários de cães orquiectomizados, avaliados por sondas fluorescentes, tempos T0 a T90 minutos pós-criopreservação $(\chi$ \pm EMP)

\begin{tabular}{lcccc}
\hline & \multicolumn{4}{c}{ Integridade da membrana plasmática (\%) } \\
\hline Técnicas/Tempo & T0 & T30 & T60 & T90 \\
FL & $33,7 \pm 17,8 \mathrm{aA}$ & $29,6 \pm 14,7 \mathrm{aA}$ & $28,2 \pm 16 \mathrm{aA}$ & $26,7 \pm 15 \mathrm{aA}$ \\
FR & $32,1 \pm 19,3 \mathrm{aA}$ & $29,6 \pm 17,8 \mathrm{aA}$ & $31 \pm 21,1 \mathrm{aA}$ & $29,2 \pm 18,5 \mathrm{aA}$ \\
\hline
\end{tabular}

Letras minúsculas diferentes na mesma linha indicam diferença estatística dentro de cada técnica, de acordo com o tempo $(\mathrm{P}<0,05)$. Letras maiúsculas diferentes na mesma coluna indicam diferença estatística entre as técnicas, de acordo com o tempo $(\mathrm{P}<0,05)$.

Em um estudo realizado após a conservação de espermatozoides epididimários de cães por um período de até 72 horas na temperatura de $4^{\circ} \mathrm{C}$, não foram observadas alterações na integridade das membranas plasmática e acrossomal. Contudo, obteve-se queda progressiva de motilidade espermática ao longo do período de conservação, nos tempos zero, 24, 48 e 72 horas (Tittarelli et al., 2006). Bustamante (2019) também afirma que a membrana plasmática de espermatozoides epididimários se manteve íntegra após 24 horas sob temperatura de $4^{\circ} \mathrm{C}$ a $6^{\circ} \mathrm{C}$.

Em outra pesquisa, com o intuito de prolongar ainda mais o tempo de conservação dos epidídimos, os autores mantiveram-nos por até oito dias em temperatura de $4^{\circ} \mathrm{C}$, e observaram diminuição da motilidade espermática. Em contrapartida, mesmo após esse longo período de refrigeração, foi possível observar células espermáticas com membrana plasmática intacta e com capacidade de ligação à zona pelúcida (Yu e Leibo, 2002). Durante as primeiras 48 horas de refrigeração, as membranas espermáticas tendem a manter sua integridade (Ponglowhapan et al., 2006).

A literatura ainda é escassa sobre a integridade de espermatozoides após a criopreservação, mas é sabido que essas células tendem a se danificar mais rapidamente com o processo da crioinjúria (Hewitt et al., 2001), sendo relevantes mais estudos sobre as melhores técnicas utilizadas para a recuperação dos espermatozoides epididimários de cães; o diluidor e a quantidade adequada para essa diluição; e o tempo necessário para o processo de refrigeração, congelação e descongelação. Esses são fatores que podem otimizar a conservação, a recuperação e a longevidade das células espermáticas.

\section{CONCLUSÃO}

Conclui-se que as técnicas de recuperação de espermatozoides epididimários de cães recentemente castrados, testadas neste trabalho, podem ser utilizadas para a refrigeração e criopreservação de sêmen.

\section{REFERÊNCIAS}

ÁlVAREZ, M.; TAMAYO-CANUL, J.; MARTÍNEZ-RODRÍGUEZ, C. et al. Specificity of the extender used for freezing ram sperm depends of the spermatozoa source (ejaculate, electroejaculate or epididymis). Anim. Reprod. Sci., v.132, p.145-154, 2012.

AXNÉR, E.; HOLST, B.S.; LINDE-FORSBERG, C. Morphology of spermatozoa in the cauda epididymis before and after electroejaculation and a comparison with ejaculated spermatozoa in the domestic cat. Theriogenology, v.50, p.973-979, 1998.

BATISTA, M.; VILAR, J.; ROSARIO, I.; TERRADAS, E. Influence of different anaesthetic protocols over the sperm quality on the fresh, chilled $\left(4^{\circ} \mathrm{C}\right)$ and frozen thawed epididymal sperm samples in domestic dogs. Reprod. Domest. Anim., v.51, p.758-765, 2016.

BENÍTEZ-GONZÁLEZ E.; CHAMBA-OCHOA H.; LUZÓN-CEVALLOS F.; SÁNCHEZCARRILlO J. Comparative evaluation of two methods of spermatic recovery of post-mortem bovine epididymis. Abanico Vet., v.8, p.59-74. 2018.

BERGO, L.C.F. Comparação de técnicas de recuperação de espermatozoides epididimários em cães. 2018. 36f. (Doutorado em Medicina Veterinária) - Universidade Federal de Viçosa, Viçosa, MG. 
BERGSTEIN-GALAN， T.G.; WEISS， R.R.; KOZICKI, L.E.; BICUDO, S.D. Espermatozoides epididimários: refrigeração e criopreservação. Rev. Bras. Reprod. Anim., v.41, p.659-664, 2017.

BLOM, E. Ultrastructure of some characteristic sperm defects and a proposal for a new classification of bull spermiogram. Nord. Vet. Med., v.25, p.383-391, 1973.

BUSTAMANTE, L.R.C. Seleção espermática em amostras ejaculadas e epididimárias de cães domésticos e efeito da adição de fluido prostático. 2019. 73f. (Doutorado em Medicina Veterinária) - Universidade Federal de Viçosa, Viçosa, MG.

DE LEEUW, F.E.; COLENBRANDER, B.; VERKLEIJ, A.J. The role membrane damage plays in cold shock and freezing injury. Reprod. Domest. Anim., v.1, p.95-104, 1991.

GRANEMANN, L.C. Avaliação comparativa do sêmen eqüino colhido com vagina artificial e por lavado intraluminal da cauda do epidídimo pósorquiectomia. 2006. 48f. Dissertação (Mestrado em Ciências Veterinárias) - Universidade Federal do Paraná, Curitiba, PR.

HARRISON, R.A.P.; VICKERS, S.E. Use of fluorescent probes to assess membrane integrity in mammalian spermatozoa. J. Reprod. Fertil., v.88, p.343-352, 1990.

HEWITT, D.A.; LEAHY, R.; SHELDON, I.M.; ENGLAND, G.C.W. Cryopreservation of epididymal dog sperm. Anim. Reprod. Sci., v.67, p.101-111, 2001.

HISHINUMA, M.; SEKINE, J. Evaluation of membrane integritiy of canine epididymal spermatozoa by short hipoosmotic swelling test with ultrapure water. J. Vet. Med. Sci., v.65, p.817-820, 2003.

HORI, T.; ATAGO, T.; KOBAYASHI, M.; KAWAKAMI, E. Influence of different methods of collection from the canine epididymides on post-thaw caudal epididymal sperm quality. $J$. Vet. Med. Sci., v.77, p.625-630. 2015.

HORI, T.; UEHARA, Y.; KAWAKAMI, E.; TSUTSUI, T. Influence of the time between removal and cooling of the canine epididymis on post-thaw caudal epididymal sperm quality. $J$. Vet. Med. Sci., v.71, p.811-815, 2009.
LIMA, D.B.C.; SILVA, T.F.P.; AQUINO CORTEZ, A. et al. Recovery of sperm after epididymal refrigeration from domestic cats using ACP-117c and Tris extenders. Arq. Bras. Med. Vet. Zootec., v.68, p.873-881, 2016.

MANUAL para exame andrológico e avaliação de sêmen animal. 3.ed. Belo Horizonte: CBRA, 2013. 104p.

MARTINEZ-PASTOR, F.; GARCIA-MACIAS, V.; ALVAREZ, M. et al. Comparison of two methods for obtaining spermatozoa from the cauda epididydimis of Iberian red deer. Theriogenology, v.65, p.471-485.2006.

MARTINS, M.I.M. Perspectivas da aplicação comercial de biotecnologias envolvendo espermatozoides obtidos de epidídimo de cães e gatos. Rev. Bras. Reprod. Anim., v.31, p.115-118, 2007.

MELO EVANGELISTA, L.S.; CARVALHO, Y.N.T.; CASTELO BRANCO, M.A. et al. Avaliação in vitro do sêmen criopreservado de cães naturalmente infectados por Leishmania sp. Arq. Bras. Med. Vet. Zootec., v.68, p.651-657, 2016.

MOTA FILHO, A.C.; SILVA, H.V.R.; NUNES, T.G.P. et al. Cryopreservation of canine epididymal sperm using ACP-106c and TRIS. Cryobiology, v.69, p.17-21, 2014.

MOTA FILHO, A.C.; SILVA, H.V.R.; FREITAS, L.A. et al. Refrigeração do epidídimo canino a $4{ }^{\circ} \mathrm{C}$ e recuperação dos espermatozoides epididimários utilizando ACP-106c. Pesq. Vet. Bras., v.9, p.1155-1160, 2013.

MOTA FILHO, A.C.; SILVA, L.D.M. Recuperação e conservação de espermatozoides epididimários de mamíferos. Acta Vet. Bras., v.6, p.1-8, 2012.

PAULA, T.A.R.; SILVA, T.; BERGO, L.; CARAZO, L.R.B.; MATEUS, L. Effect of different media used during the collection of spermatozoa from the tail of the epididymis in the dog. In: CONGRESS EUROPEAN VETERINARY SOCIETY FOR SMALL ANIMAL REPRODUCTION, 21., 2018, Venice. Proceedings... Venice: EVSSAR, 2018.

PENNACCHISAVI, P.A.; MOTHEO, T.F.; NAKAGHI, L.C.P.; BUTTLER, E.P.; VICENTE, W.R.R. Técnica modificada de compressão do ducto deferente e cauda do epidídimo para 
obtenção de espermatozoides caninos. Rev. Investigação, v.14, p.1-7, 2015.

PLANT, T.; ZELEZNIK, A. Knobil \& Neill's physiology of reproduction. 4.ed. USA: Amsterdam: Elsevier, 2014. v.1, 2684p.

PONGLOWHAPAN, S.; CHATDARONG, K.; SIRIVAIDYAPONG, S.; LOHACHIT, C. Freezing of epididymal spermatozoa from dogs after cool storage for 2 or 4 days. Theriogenology, v.66, p.1633-1636. 2006.

RIZZOTO, G.; MOREIRA, F.; VARELA JUNIOR, A.S.; DODE, M.E.B. et al. Efeitos da adição de glicerol e etilenoglicol associados sobre parâmetros de viabilidade espermática na criopreservação de sêmen canino. PubVet, v.8, p.2675-2805, 2014.
STRÖM, B.; ROTA, A.; LINDE-FORSBERG, C. In vitro characteristics of canine spermatozoa subjected to two methods of cryopreservation. Theriogenology, v.48, p.247-256, 1997.

TITTARELLI, C.; SAVIGNONE, C.A.; ARNAUDÍN, E. et al Effect of storage media and storage time on survival of spermatozoa recovered from canine and feline epididymides. Theriogenology, v.66, p.1637-1640, 2006.

VARESI, S.; VERNOCCHI, V.; MORSELLI, M.G.; LUVONI, G.C. DNA integrity of fresh and frozen canine epididymal spermatozoa. Reprod. Biol., v.14, p.257-261, 2014.

YU, I.; LEIBO, S.P. Recovery of motile, membrane-intact spermatozoa from canine epididymides stored for 8 days at $4{ }^{\circ} \mathrm{C}$. Thereogenology, v.57, p.1179-1190, 2002. 